\title{
The effect of temporary closure and enhanced terminal disinfection using aerosolized hydrogen peroxide of an open- bay intensive care unit on the acquisition of extensively drug-resistant Acinetobacter baumannii
}

Rima Moghnieh ${ }^{1,2^{*}}$, Hani Tamim³ ${ }^{3}$, Marwa Jadayel ${ }^{4}$, Dania Abdallah ${ }^{5}$, Rasha Al-Kassem ${ }^{6}$, Hind Kadiri ${ }^{7}$, Hani Hafez ${ }^{7}$, Salam Al-Hassan ${ }^{6}$, Lina Ajjour ${ }^{6}$, Rawad Lakkis ${ }^{8}$, Tamima Jisr ${ }^{9}$, Nadia-Lara Samaha ${ }^{10}$ and Nicholas Haddad ${ }^{11}$

\begin{abstract}
Background: At Makassed Hospital's open-bay intensive care unit (ICU), enhanced terminal disinfection (ETD) using hydrogen peroxide $\left(\mathrm{H}_{2} \mathrm{O}_{2}\right)$ was performed without a predefined schedule in extensively-drug-resistant Acinetobacter baumannii (XDR-AB) outbreaks. In this study, we aimed to check for the value of the temporary closure of the ICU and the use of ETD with aerosolized $\mathrm{H}_{2} \mathrm{O}_{2}$ and $\mathrm{Ag}^{+}$on minimizing the rate of XDR-AB acquisition in patients admitted to the ICU of our facility, which might consequently help us determine the optimal schedule for such procedure in this unit.

Methods: This is a retrospective medical file review of patients admitted to the ICU between January 2016 and May 2018. We divided this period into numerical weeks (NW) after each closure and ETD episode. Risk factors of acquisition (RFA) were determined by comparing the characteristics of patients who acquired XDR-AB to those who didn't. The proportion of patients residing in each NW was included in the RFA analysis.

Results: Out of 335 patients, 13\% acquired XDR-AB. The overall incidence of XDR-AB acquisition was 14.6 cases/1000 patient days. RFA were XDR-AB contact pressure $\geq 3$ days [Odds Ratio $(\mathrm{OR})=9.86,95 \%$ Confidence Interval $(\mathrm{Cl})(3.65-$ 26.64), $P<0.0001)]$, mechanical ventilation $[\mathrm{OR}=4.99,95 \% \mathrm{Cl}(1.76-14.15), P=0.002)]$, and having a wound $[\mathrm{OR}=3.72$, $95 \% \mathrm{Cl}(0.99-13.96), P=0.05)]$. Patients who stayed during NW 7,11 and 14 were at risk of acquisition where the odds significantly increased by $6.5,9.7$ and 14.4 folds respectively $(P=0.03,0.01$, and 0.01 , respectively). We considered NW 7 as the most suitable time for temporary closure of the ICU and ETD with aerosolized $\mathrm{H}_{2} \mathrm{O}_{2}$.

(Continued on next page)
\end{abstract}

\footnotetext{
* Correspondence: moghniehrima@gmail.com

'Division of Infectious Diseases, Department of Internal Medicine, Makassed General Hospital, Beirut, Lebanon

2Division of Infectious Diseases, Hôtel Dieu de France, Beirut, Lebanon

Full list of author information is available at the end of the article
}

(c) The Author(s). 2020 Open Access This article is licensed under a Creative Commons Attribution 4.0 International License, which permits use, sharing, adaptation, distribution and reproduction in any medium or format, as long as you give appropriate credit to the original author(s) and the source, provide a link to the Creative Commons licence, and indicate if changes were made. The images or other third party material in this article are included in the article's Creative Commons licence, unless indicated otherwise in a credit line to the material. If material is not included in the article's Creative Commons licence and your intended use is not permitted by statutory regulation or exceeds the permitted use, you will need to obtain permission directly from the copyright holder. To view a copy of this licence, visit http://creativecommons.org/licenses/by/4.0/ The Creative Commons Public Domain Dedication waiver (http://creativecommons.org/publicdomain/zero/1.0/) applies to the data made available in this article, unless otherwise stated in a credit line to the data. 
(Continued from previous page)

Conclusion: Contact pressure, mechanical ventilation, and presence of a wound were RFA of XDR-AB. Temporary closure of the ICU with ETD using aerosolized $\mathrm{H}_{2} \mathrm{O}_{2}$ decreased the rate of XDR-AB acquisition, yet this effect fades away with time. The ETD was shown to be most efficiently done when repeated every 7 calendar weeks in our open-bay ICU as part of a prevention bundle.

Keywords: Enhanced terminal disinfection, Hydrogen peroxide, Extensively drug-resistant Acinetobacter baumannii, Intensive care unit, Open-bay, Contact pressure, Lebanon

\section{Background}

With the turn of the century, the upsurge of antimicrobial resistance (AMR) has become a worldwide public health threat and its control has become a global priority [1]. Lebanon is at risk for this alarming situation where carbapenem-resistant Gram-negative organisms are widespread in hospitals and have reached the community setting [2-5]. Some of these organisms are even resistant to almost all available antibiotics $[6,7]$. Consequently, controlling their spread in both settings is of critical importance.

Acinetobacter baumannii is one of the most troublesome pathogens in hospitals causing nosocomial infections and it is associated with high mortality rates, especially among critically ill patients $[8,9]$. Of particular concern is its prolonged survival in the hospital environment through its resistance to desiccation thus causing sustained outbreaks [10]. Its inherent and acquired mechanisms of resistance to multiple antibiotics render it a difficult-to-treat pathogen $[8,9]$. Carbapenem-resistant or extensively-drug resistant Acinetobacter baumannii (XDR-AB) is already endemic in many hospitals in Lebanon [2-6] and pan-drug resistant isolates that are resistant to colistin are progressively appearing in hospitals $[7,11]$.

Transmission of A. baumannii from the inanimate hospital environment to patients has been well documented $[10,12,13]$. As part of an infection prevention bundle, enhanced terminal environmental disinfection (ETD) is one of the modalities that have been effective in decreasing the spread of these organisms [14]. The most common nontouch techniques of ETD are using ultraviolet light or hydrogen peroxide vapor or aerosol [15].

Makassed General Hospital (MGH), a tertiary care hospital in Beirut, has an open-bay intensive care unit (ICU). As of 2011, XDR-AB acquisition has been reported at an average of 1 case per 80 patient days in this unit (non-published hospital surveillance data). ETD using aerosolized hydrogen peroxide $\left(\mathrm{H}_{2} \mathrm{O}_{2}\right)$ and Silver ions $\left(\mathrm{Ag}^{+}\right)$is performed in this ICU and in rooms of the hospital that have been previously occupied by patients colonized or infected patients with pan-drug and extensively-drug resistant bacteria, amongst which is A. baumannii. In the ICU, there is no predefined schedule for ETD where it is performed upon the request of physicians. It is also subject to convenience, based on bed occupancy as ETD necessitates evacuation of the unit for at least $24 \mathrm{~h}$. In this study, we primarily aimed to check for the value of temporary closure of the ICU along with ETD using aerosolized $\mathrm{H}_{2} \mathrm{O}_{2}$ and $\mathrm{Ag}^{+}$on minimizing the rate of $\mathrm{XDR}-\mathrm{AB}$ acquisition in patients admitted to the ICU of our facility, which might consequently help us determine the optimal schedule for such procedure in this unit as part of an infection prevention bundle. A secondary endpoint was to check for the presence of risk factors of XDR-AB acquisition, related to patient clinical characteristics, the use of invasive devices, and the use of antimicrobial agents. Risk factor identification and mitigation are critical pillars in decreasing the transmission of XDR-AB and these factors will be additionally controlled for in the analysis model.

\section{Methods}

\section{Setting and study design}

This is a retrospective study conducted at MGH, a 186bed university hospital and tertiary care referral center, with a 5-bed open-bay mixed medical and surgical adult ICU. The study period extended from January 2016 to May 2018. It included data from patient charts admitted to the ICU during the study period. Clinical, microbiological and radiographic information were retrieved from electronic medical records. Other information regarding the number of admissions to the ICU, bed census and number of patient days (PD) were obtained from the nursing head office. The dates of terminal cleaning during the study were retrieved from the hospital administration records. The hospital's Institutional Review Board committee approved this study and patient consent was waived due to its retrospective nature.

\section{Infection control considerations}

Active microbiological screening for patients' colonization status and acquisition of carbapenem-resistant organisms was performed during the study period in the ICU. Specimens from the throat, axillae, urine, and perineal area were routinely cultured, in addition to sputum and/or skin lesion (aspirate/biopsy/swab) when applicable. These cultures were secured upon ICU admission and on a weekly 
basis, as long as the patient was still there and whenever the clinical situation necessitated. The nurse-to patient ratio in the ICU was 2 registered nurses and 3 practical nurses to 5 patients (2:3:5).

Infection control measures were applied according to the Centers for Disease Control and Prevention (CDC) guidelines for the control of spread of extensively-drug resistant organisms in healthcare settings [16]. Daily ICU environmental cleaning and disinfection, with the beds occupied, was performed using an alcohol-based disinfectant and a quaternary ammonium containing disinfectant. As mentioned previously, ETD was performed after evacuating the unit according to bed occupancy convenience, the ICU/Infectious Disease physician request and/or Infection Control team recommendations, without a predefined schedule. It was performed using $6 \%$ aerosolized $\mathrm{H}_{2} \mathrm{O}_{2}$ with 60 part-per-million Silver ions $\left(\mathrm{Ag}^{+}\right)$(BioGienie ${ }^{\mathrm{Tm}}$ H1000 Version 1, HHYGIENICS ${ }^{\mathrm{mm}}$ BIOSECURITY, Hampshire, United Kingdom).

\section{Microbiological identification}

The identification of bacteria from all types of cultures was performed according to standard microbiological procedures. Surveillance cultures targeted carbapenemresistant Gram-negative bacilli, such as $A$. baumannii, by using MacConkey agar with a $10 \mu \mathrm{g}$ ertapenem disc and a $10 \mu \mathrm{g}$ meropenem disc. All microbiological methods were consistent with the Clinical and Laboratory Standards Institute (CLSI) guidelines and antimicrobial susceptibility was determined using the CLSI breakpoints of each corresponding year [17]. XDR-AB was considered resistant to at least one agent in all but two or fewer antimicrobial categories (i.e. bacterial isolates remain susceptible to only one or two categories) [18]. In this study, XDR-AB remained susceptible to polymyxin $\mathrm{E}$ (colistin) and tigecycline.

\section{Grouping of patients}

In this study, we divided ICU patients to three groups based on their acquisition of XDR-AB:

- Group 1 or test group included patients who acquired XDR-AB during their stay at least $48 \mathrm{~h}$ after admission to the ICU or until 1 week after discharge from the ICU. This acquisition was defined as the isolation of the organism for the very first time from any body site in the patient's history. All subsequent positive XDR-AB cultures from the same patient were considered duplicate and were excluded from the analysis.

- -Group 2 or control group included patients admitted to ICU during the study period and who did not show any evidence of XDR-AB acquisition at anytime.
- -Group 3 included patients who:

1. Had positive XDR-AB cultures recovered from any body site within the first $48 \mathrm{~h}$ of admission to the ICU,

2. Had evidence of colonization and /or infection with $\mathrm{XDR}-\mathrm{AB}$ from a previous admission to our facility during 1 year prior to the study period,

3. Were admitted to our ICU or any other ICU in the past 3 months prior to the study period,

4. Were transferred from or admitted to another acute-care or long-term healthcare facility within the past 3 months prior to the study period.

\section{Potential risk factors}

Patients in Groups 1 and 2 only were included in the analysis of risk factors for XDR-AB acquisition. The parameters in question were age, gender, reason for ICU admission, history of intra-abdominal surgery, mechanical ventilation and its duration, use of bilevel positive airway pressure (BiPAP), central venous catheterization and its duration, urinary catheterization and its duration, use of other types of catheters with the duration, presence of wounds or pressure ulcers, the use of vasopressors, the needs of blood transfusion, duration of ICU stay, Acute Physiologic Assessment and Chronic Health Evaluation II (APACHE II) score, Charlson Comorbidity Index (CCI) score, carbapenem and/or piperacillin tazobactam intake with the duration, and XDR-AB contact pressure days. For each patient, contact pressure is a way to measure the extent of contact with colonized or infected patients during a certain bed occupancy time, that is to determine the threat of XDR-AB acquisition exerted by other colonized or infected patients. Contact pressure, expressed in days, is calculated by adding up the number of days that were spent in the vicinity of a colonized or infected patient, i.e. in the same ICU bay or sharing the same room outside ICU in this study [2]. Patients in Group 3 were not included in the risk factor analysis; however, they were included in the calculation of XDR-AB contact pressure days for each patient in Groups 1 and 2. For all time-dependent parameters in Group 1, we only took into account the duration preceding the date of XDR-AB acquisition.

\section{The effect of temporary closure and ETD using aerosolized $\mathrm{H}_{2} \mathrm{O}_{2}$ and $\mathrm{ag}^{+}$on XDR-AB acquisition}

We obtained the dates of ETD from the IC department, and, accordingly, we divided the study period into numerical week, following each event of ETD. The first week after each ETD was considered 'Week 1', followed by 'Week 2', etc. In order to determine the risk of XDR- 
$\mathrm{AB}$ acquisition in patients residing in the ICU during each numerical week, we recorded the number of patients who stayed in the ICU in each week as per their admission and discharge dates. Patients were counted more than once depending on their duration of stay with respect to the numerical weeks after ETD. The numerical weeks were considered potential risk factors to be included in the statistical analysis between Groups 1 and 2. Patients in Group 3 were excluded from this analysis.

\section{Incidence of XDR-AB acquisition with time (numerical weeks after ETD)}

The number of PD in the ICU was calculated by counting the patient census at the same time each day. We plotted the number of PD (Y-axis) against each numerical week ('Week 1, 2, 3, etc.') (X-axis). We also plotted the incidence rate of XDR-AB acquisition in Group 1 (the number of patients who acquired XDR-AB per 1000 $\mathrm{PD})$ (Y-axis) against each numerical week (X-axis), taking into account their first acquisition. Each patient was counted once per each numerical week depending on the date of acquisition only and it was not cumulative. Only the numerical weeks with PD $>50$ were included in the plot of XDR-AB incidence and in the analysis of the relation between the numerical week after ETD and the probability of acquiring XDR-AB in ICU.

\section{Statistical analysis}

Statistical analysis was performed by the Statistical Package for the Social Sciences program (SPSS) version 24 (SPSS, Chicago, IL, USA). All p-values were two tailed and $P<0.05$ was considered statistically significant. Categorical variables were compared to assess any significance using chi-square test and were presented as frequency (\%). Bivariate analysis of all studied parameters was performed between groups 1 and 2 and those with $P<0.05$ were included in the multivariate logistic regression. Odds ratio (OR) and 95\% confidence interval (CI) were reported to indicate the impact and significance of each variable in the multivariate model. The first numerical week (PD > 50) where there was a statistically significant difference between the groups 1 and 2 in the bivariate model was recorded. This week and all numerical weeks thereafter were included in the multivariate analysis of independent risk factors for $\mathrm{XDR}-\mathrm{AB}$ acquisition, while controlling for other factors. In the multivariate analysis, the first numerical week with an $\mathrm{OR}>2$ and a $p$-value $<0.05$ was considered the optimal week to routinely perform ETD.

\section{Results}

\section{Description of patient characteristics}

During the study period, 335 patients were admitted to ICU, with a total of 3083 patient days. The baseline clinical characteristics of the study population, and the studied risk parameters are listed in Table 1 . The majority of the patients were above 75 years of age (154/335 patients, $46 \%)$ and $51 \%$ were males (170/335). The two most common causes of ICU admission were respiratory disorders and sepsis (34.9 and $30.4 \%$, respectively). One third of the patients had an APACHE II score of 20 and above and $72.8 \%$ had a CCI score more than or equal to 4. Mechanical ventilation was required in $50.4 \%$ of the cases, $31.3 \%$ of the patients had central lines and $91.3 \%$ had urinary catheters. Vasopressors were administered in $51.6 \%$ of the patients and blood transfusion in $29.3 \%$. Carbapenems were prescribed in $58.2 \%$ of the cohort and piperacillin-tazobactam in $35.5 \%$. The distribution of patients per group was as such: $13.1 \%$ for Group 1 (44/335 patients), 84.2\% for Group 2 (282/335 patients) and $2.7 \%$ for Group 3 (9/335 patients) (Table 1).

\section{Effect of temporary closure and ETD on XDR-AB acquisition measured by the variation in its incidence rate during the numerical weeks}

Temporary closure of ICU and ETD using aerosolized $\mathrm{H}_{2} \mathrm{O}_{2}$ and $\mathrm{Ag}^{+}$was performed 8 times during the study period and the number of numerical weeks reached 38 (Fig. 1). During the entire study period, the overall incidence of XDR-AB acquisition reached 14.6 cases per $1000 \mathrm{PD}$. Regarding the variation in the incidence rate of XDR-AB acquisition per numerical week (Fig. 2), a linear increasing trend was observed from numerical Week 1 to Week 16 after ETD, where it was 5.4 cases/ 1000 PD in Week 1, 11 cases/1000 PD in Week 7, and it significantly increased to 35.7 cases/1000 PD in Week 16 $(P=0.003)$. Weeks 17 to 38 after ETD were not included due to the low number of PD ( $<50$ days).

\section{Risk factors for the acquisition of XDR-AB}

Through bivariate analysis, there was a statistically significant difference in several parameters between Groups 1 and 2, respectively, thus favoring XDR-AB acquisition in the following: Presence of a wound $(13.6 \%$ vs. $3.5 \%$, $P=0.01)$, mechanical ventilation $(81.8 \%$ vs. $44.7 \%, P<$ 0.0001 ), placement of a urinary catheter for more than or equal to 5 days $(79.5 \%$ vs. $32.6 \%, P=0.002)$, receipt of carbapenems for more than or equal to 4 days $(56.8 \%$ vs. $34.4 \%, P=0.004)$, receipt of piperacillin-tazobactam $(52.3 \%$ vs. $33 \%, P=0.01)$, ICU stay of 4 or more days ( $84.1 \%$ vs. $41.8 \%, P<0.0001)$, and XDR-AB contact pressure of 3 or more days $(68.2 \%$ vs. $18.4 \%, P=0.0001)$ (Table 1).

After controlling for confounding factors, the multivariate model showed that mechanical ventilation increased the risk of XDR-AB acquisition by almost 5 times $[\mathrm{OR}=4.99,95 \%$ CI $(1.76-14.15), \quad P=0.002)]$ (Table 2). Patients with XDR-AB contact pressure for 3 
Table 1 Bivariate analysis of risk factors for extensively drug-resistant Acinetobacter baumannii acquisition in the open-bay intensive care unit during the study period

\begin{tabular}{|c|c|c|c|c|c|}
\hline Characteristics & $\begin{array}{l}\text { Total } \\
(\boldsymbol{N}=335 \text { patients) (\%) }\end{array}$ & $\begin{array}{l}\text { Group } 1 \\
(\boldsymbol{N}=44 \text { patients) }(\%)\end{array}$ & $\begin{array}{l}\text { Group } 2 \\
(\boldsymbol{N}=282 \text { patients })(\%)\end{array}$ & $\mathbf{P}$ (Group 1 vs. Group 2) & $\begin{array}{l}\text { Group } 3 \\
(\boldsymbol{N}=9 \text { patients) }(\%)\end{array}$ \\
\hline \multicolumn{6}{|l|}{ Age (years) } \\
\hline$\leq 45$ & 49 (14.6) & $4(9.1)$ & $44(15.6)$ & 0.23 & $1(11.1)$ \\
\hline$>45$ and $<75$ & $132(39.4)$ & $15(34.1)$ & $115(40.8)$ & & $2(22.2)$ \\
\hline$\geq 75$ & $154(46.0)$ & $25(56.8)$ & $123(43.6)$ & & $6(66.7)$ \\
\hline Gender & & & & 0.29 & \\
\hline Female & $165(49.3)$ & $25(56.8)$ & $136(48.2)$ & & $4(44.4)$ \\
\hline Male & $170(50.7)$ & $19(43.2)$ & $146(51.8)$ & & $5(55.6)$ \\
\hline \multicolumn{6}{|l|}{ Cause of ICU admission } \\
\hline Respiratory problem & $117(34.9)$ & $15(34.1)$ & $97(34.4)$ & 0.97 & $5(55.6)$ \\
\hline Septic shock & $102(30.4)$ & $16(36.4)$ & $83(29.4)$ & 0.35 & $3(33.3)$ \\
\hline Post-surgical Observation & $38(11.3)$ & $5(11.4)$ & $33(11.7)$ & 0.95 & $0(0.0)$ \\
\hline Other causes & $78(23.3)$ & $8(18.2)$ & $69(24.5)$ & 0.36 & $1(11.1)$ \\
\hline Intra-abdominal surgery & $23(6.9)$ & $4(9.1)$ & $19(6.8)$ & 0.53 & $0(0.0)$ \\
\hline Pressure ulcer & $51(15.2)$ & $9(20.5)$ & $38(13.5)$ & 0.22 & $4(44.4)$ \\
\hline Wound & $17(5.1)$ & $6(13.6)$ & $10(3.5)$ & 0.01 & $1(11.1)$ \\
\hline Urinary catheter & $306(91.3)$ & $44(100.0)$ & $253(89.7)$ & 0.02 & $9(100.0)$ \\
\hline$<5 d$ & $176(52.5)$ & $10(22.7)$ & $163(57.8)$ & $<0.0001$ & $3(33.3)$ \\
\hline$\geq 5 d$ & $159(47.5)$ & $34(77.3)$ & $119(42.2)$ & & $6(66.7)$ \\
\hline Mechanical ventilation & $169(50.4)$ & $36(81.8)$ & $126(44.7)$ & $<0.0001$ & $7(77.8)$ \\
\hline$<2 d$ & $204(60.9)$ & $9(20.5)$ & $190(67.4)$ & 0.002 & $5(55.6)$ \\
\hline$\geq 2 d$ & $131(39.1)$ & $35(79.5)$ & $92(32.6)$ & & $4(44.4)$ \\
\hline BiPAP use at hospital & $83(24.8)$ & $11(25.0)$ & $70(24.8)$ & 0.98 & $2(22.2)$ \\
\hline BiPAP use at home & $25(7.5)$ & $2(4.5)$ & $23(8.2)$ & 0.55 & $0(0.0)$ \\
\hline Central line & $105(31.3)$ & $19(43.2)$ & $82(29.1)$ & 0.06 & $4(44.4)$ \\
\hline Kemal catheter & $33(9.9)$ & $6(13.6)$ & $27(9.6)$ & 0.42 & $0(0.0)$ \\
\hline Hemodialysis in ICU & $44(13.1)$ & $5(11.4)$ & $39(13.8)$ & 0.66 & $0(0.0)$ \\
\hline Use of vasopressors & $173(51.6)$ & $27(61.4)$ & $141(50.0)$ & 0.16 & $5(55.6)$ \\
\hline \multicolumn{6}{|l|}{ APACHE II score } \\
\hline$<20$ & $220(65.7)$ & $27(61.4)$ & $189(67.0)$ & 0.46 & $4(44.4)$ \\
\hline$\geq 20$ & $115(34.3)$ & $17(38.6)$ & $93(33.3)$ & & $5(55.6)$ \\
\hline Between 20 and 29 & $86(25.7)$ & $15(34.1)$ & $68(73.1)$ & 0.35 & $3(33.3)$ \\
\hline$\geq 30$ & $29(8.7)$ & $2(4.5)$ & $25(26.9)$ & & $2(22.2)$ \\
\hline \multicolumn{6}{|l|}{$\mathrm{CCl}$ score } \\
\hline 0 & $38(11.3)$ & $3(6.8)$ & $34(12.1)$ & 0.047 & $1(11.1)$ \\
\hline $1-2$ & $31(9.3)$ & $4(9.1)$ & $26(9.2)$ & & $1(11.1)$ \\
\hline $3-4$ & $75(22.4)$ & $12(27.3)$ & $61(21.6)$ & & $2(22.2)$ \\
\hline $5-6$ & $97(29.0)$ & $19(43.2)$ & $76(27.0)$ & & $2(22.2)$ \\
\hline $7-8$ & $62(18.5)$ & $6(13.6)$ & $55(19.5)$ & & $1(11.1)$ \\
\hline$>8$ & $32(9.6)$ & $0(0.0)$ & $30(10.6)$ & & $2(22.2)$ \\
\hline$<4$ & $91(27.2)$ & $12(27.3)$ & $76(27.0)$ & 0.96 & $3(33.3)$ \\
\hline$\geq 4$ & $244(72.8)$ & $32(72.7)$ & $206(73.0)$ & & $6(66.7)$ \\
\hline XDR-AB contact pressure & $147(43.9)$ & $33(75.0)$ & $113(40.1)$ & $<0.0001$ & $1(11.1)$ \\
\hline$<3 d$ & $252(75.2)$ & $14(31.8)$ & $230(81.6)$ & $<0.0001$ & $8(88.9)$ \\
\hline
\end{tabular}


Table 1 Bivariate analysis of risk factors for extensively drug-resistant Acinetobacter baumannii acquisition in the open-bay intensive care unit during the study period (Continued)

\begin{tabular}{|c|c|c|c|c|c|}
\hline Characteristics & $\begin{array}{l}\text { Total } \\
(\boldsymbol{N}=335 \text { patients })(\%)\end{array}$ & $\begin{array}{l}\text { Group } 1 \\
(\boldsymbol{N}=44 \text { patients })(\%)\end{array}$ & $\begin{array}{l}\text { Group } 2 \\
(\boldsymbol{N}=282 \text { patients) (\%) }\end{array}$ & $\boldsymbol{P}$ (Group 1 vs. Group 2) & $\begin{array}{l}\text { Group } 3 \\
(\boldsymbol{N}=9 \text { patients) (\%) }\end{array}$ \\
\hline$\geq 3 d$ & $83(24.8)$ & $30(68.2)$ & $52(18.4)$ & & $1(11.1)$ \\
\hline Blood Transfusion & $98(29.3)$ & $11(25.0)$ & $82(29.1)$ & 0.58 & $5(55.6)$ \\
\hline CAR use & $195(58.2)$ & $31(70.5)$ & $157(55.7)$ & 0.06 & $7(77.8)$ \\
\hline$<4 d$ & $208(62.1)$ & $19(43.2)$ & $185(65.6)$ & 0.004 & $4(44.4)$ \\
\hline$\geq 4 d$ & $127(37.9)$ & $25(56.8)$ & $97(34.4)$ & & $5(55.6)$ \\
\hline$<7 d$ & $246(73.4)$ & $25(56.8)$ & $216(76.6)$ & 0.005 & $5(55.6)$ \\
\hline$\geq 7 d$ & 89 (26.6) & $19(43.2)$ & $66(23.4)$ & & $4(44.4)$ \\
\hline TZP use & $119(35.5)$ & $23(52.3)$ & $93(33.0)$ & 0.01 & $3(33.3)$ \\
\hline$<4 d$ & $275(82.1)$ & $34(77.3)$ & $234(83.0)$ & 0.36 & $7(77.8)$ \\
\hline$\geq 4 d$ & $60(17.9)$ & $10(22.7)$ & $48(17.0)$ & & $2(22.2)$ \\
\hline CAR or TZP use & $259(77.3)$ & $43(97.7)$ & $207(73.4)$ & $<0.0001$ & $9(100.0)$ \\
\hline$<4 d$ & $164(49.0)$ & $12(27.3)$ & $149(52.8)$ & 0.002 & $3(33.3)$ \\
\hline$\geq 4 d$ & $171(51.0)$ & $32(72.7)$ & $133(47.2)$ & & $6(66.7)$ \\
\hline \multicolumn{6}{|l|}{ LOS in the ICU } \\
\hline$<4$ days & $175(52.2)$ & $7(15.9)$ & $164(58.2)$ & $<0.0001$ & $4(44.4)$ \\
\hline$\geq$ days & $160(47.8)$ & $37(84.1)$ & $118(41.8)$ & & $5(55.6)$ \\
\hline \multicolumn{6}{|c|}{ Number of patients who stayed in the ICU during each numerical week after ETD ${ }^{a}$} \\
\hline Week 1 & $54(16.1)$ & $6(13.6)$ & 44 (15.6) & 0.74 & $4(44.4)$ \\
\hline Week 2 & 35 (10.4) & $5(11.4)$ & $27(9.6)$ & 0.78 & $3(33.3)$ \\
\hline Week 3 & 37 (11.0) & $5(11.4)$ & $32(11.3)$ & 1.00 & $0(0.0)$ \\
\hline Week 4 & 35 (10.4) & $5(11.4)$ & $29(10.3)$ & 0.79 & $1(11.1)$ \\
\hline Week 5 & 38 (11.3) & $6(13.6)$ & $31(11.0)$ & 0.61 & $1(11.1)$ \\
\hline Week 6 & $31(9.3)$ & $8(18.2)$ & $21(7.4)$ & 0.04 & $2(22.2)$ \\
\hline Week 7 & $30(9.0)$ & $7(15.9)$ & $21(7.4)$ & 0.08 & $2(22.2)$ \\
\hline Week 8 & 38 (11.3) & $6(13.6)$ & $31(11.0)$ & 0.61 & $1(11.1)$ \\
\hline Week 9 & $36(10.7)$ & $8(18.2)$ & $26(9.2)$ & 0.11 & $2(22.1)$ \\
\hline Week 10 & $19(5.7)$ & $5(11.4)$ & $14(5.0)$ & 0.15 & $0(0.0)$ \\
\hline Week 11 & $25(7.5)$ & 7 (15.9) & $17(6.0)$ & 0.03 & $1(11.1)$ \\
\hline Week 12 & $26(7.8)$ & $8(18.2)$ & $17(6.0)$ & 0.01 & $1(11.1)$ \\
\hline Week 13 & $22(6.6)$ & $7(15.9)$ & $14(5.0)$ & 0.01 & $1(11.1)$ \\
\hline Week 14 & $20(6.0)$ & $10(22.7)$ & $10(3.5)$ & $<0.0001$ & $0(0.0)$ \\
\hline Week 15 & $17(5.1)$ & $8(18.2)$ & $9(3.2)$ & 0.001 & $0(0.0)$ \\
\hline Week 16 & $18(5.4)$ & $6(13.6)$ & $12(4.3)$ & 0.02 & $0(0.0)$ \\
\hline
\end{tabular}

$K E Y=A P A C H E$ Acute Physiology And Chronic Health Evaluation, BiPAP Bilevel Positive Airway Pressure, CAR Carbapenems, CCI Charlson Comorbidity Index, $d$ days, ETD Enhanced Terminal Disinfection, ICU Intensive Care Unit, LOS Length of Stay, TZP Piperacillin/Tazobactam, XDR-AB Extensively-drug resistant Acinetobacter bumannii

N.B

In this study, we divided ICU patients to three groups based on their acquisition of XDR A. baumannii (XDR-AB):

-Group 1 or test group included patients who acquired XDR-AB during their stay at least $48 \mathrm{~h}$ after admission to the ICU or until 1 week after discharge from the ICU.

-Group 2 or control group included patients admitted to ICU during the study period and who did not show any evidence of XDR-AB acquisition at anytime.

-Group 3 included patients who:

1. Had positive XDR-AB cultures recovered from any body site within the first $48 \mathrm{~h}$ of admission to the ICU,

2. Had evidence of colonization and /or infection with XDR-AB from a previous admission to our facility during 1 year prior to the study period,

3. Were admitted to our ICU or any other ICU in the past 3 months prior to the study period,

4. Were transferred from or admitted to another acute-care or long-term healthcare facility within the past 3 months prior to the study period.

a Patients who resided in the ICU during numerical weeks 1 to 16 were only included since the number of patient days per week was $>50$ days. Patients were counted more than once depending on their duration of stay with respect to the numerical weeks after ETD 


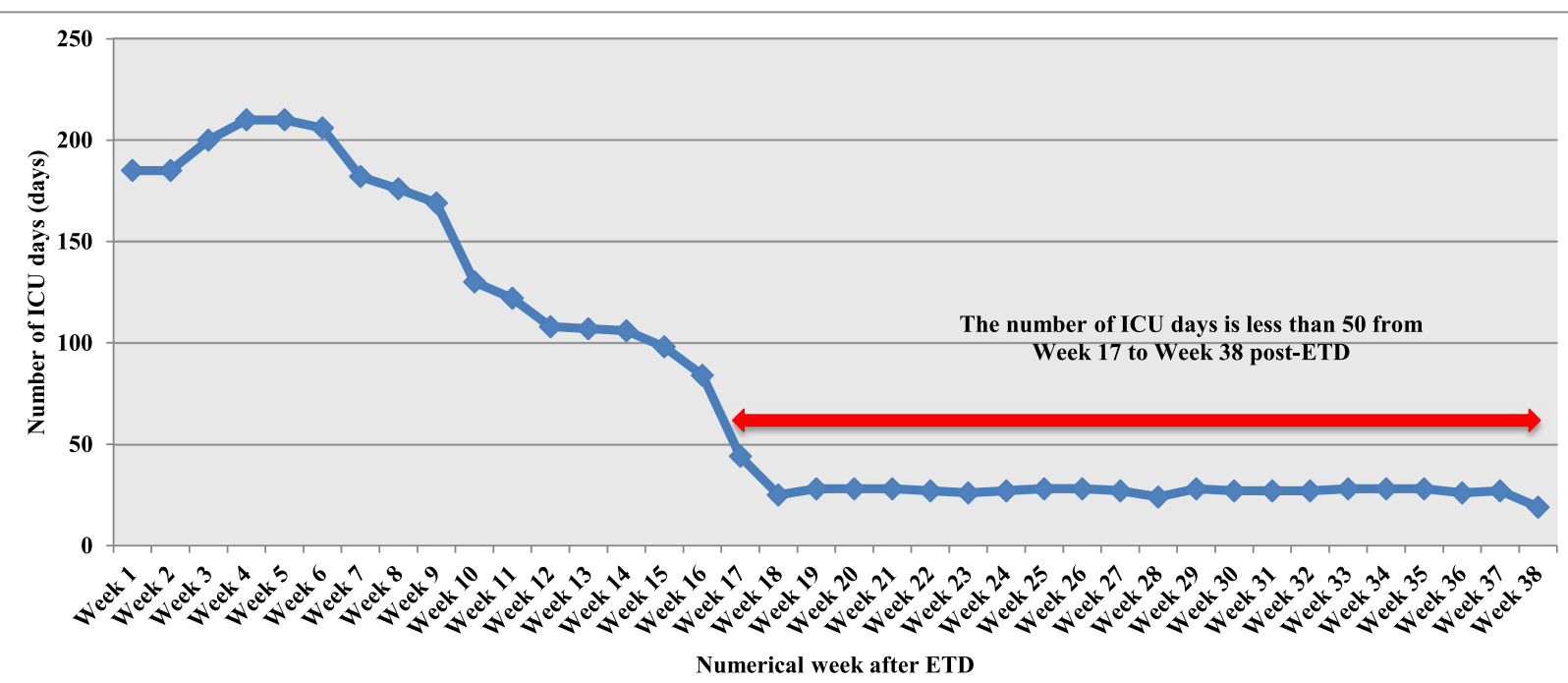

Fig. 1 The number of patient days in the open-bay intensive care unit (ICU) of our facility per each numerical week after enhanced terminal disinfection (ETD) during the study period

or more days were at the highest risk of acquisition $[\mathrm{OR}=9.86,95 \%$ CI $(3.65-26.64), P<0.0001)]$. On the other hand, the presence of a wound increased the risk of acquisition by 4 times and nearly reached statistical significance $[\mathrm{OR}=3.72,95 \% \mathrm{CI}(0.99-13.96), P=0.05)]$. Likewise, the receipt of piperacillin-tazobactam and carbapenems for more than 4 days increased the odds of
XDR-AB acquisition by almost 3 times yet did not reach statistical significance $[\mathrm{OR}=2.86,95 \%$ CI $(0.97-8.46)$, $P=0.06) ; \mathrm{OR}=2.5,95 \% \mathrm{CI}(0.43-14.36), P=0.31)$; respectively]. As for the placement of a urinary catheter for 5 or more days and ICU LOS of 4 or more days, both parameters increased the odds by 1.3 folds yet without statistical significance $[\mathrm{OR}=1.42,95 \%$ CI $(00.42-4.79)$,

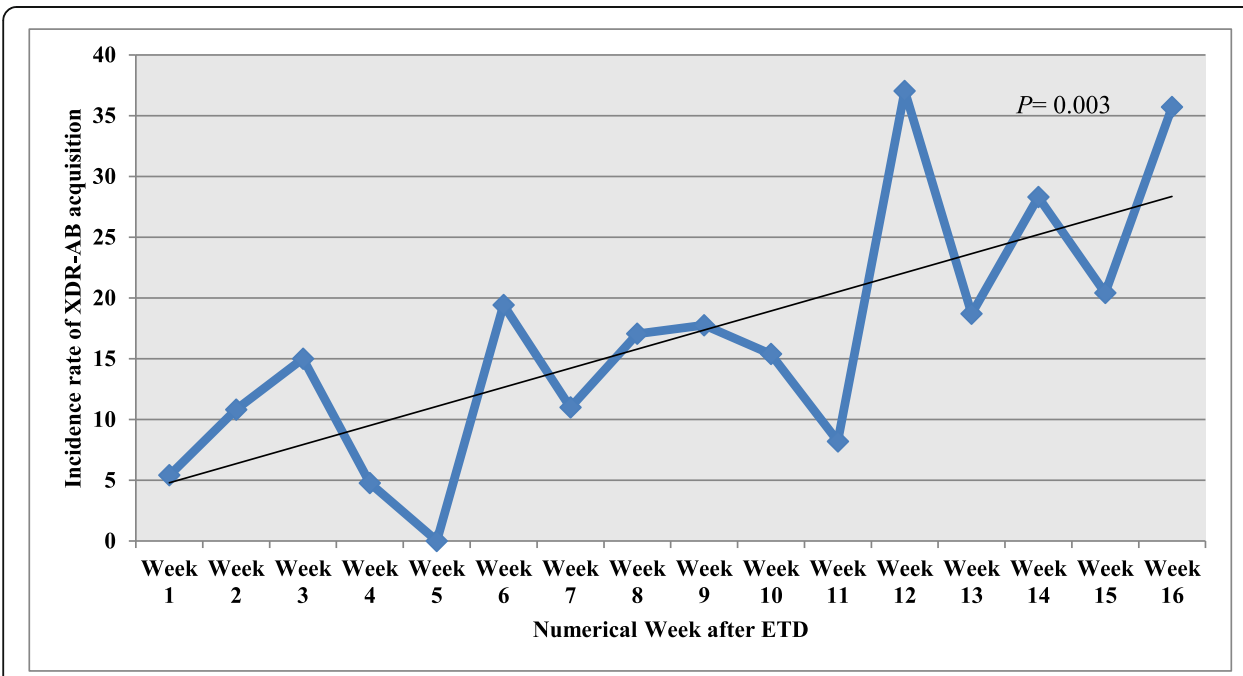

N.B. -Each patient, who resided in the ICU during the corresponding numerical week, was counted once per each numerical week depending on the date of acquisition only and it was not cumulative.

-The incidence rate of XDR-AB was calculated from Week 1 to Week 16 after ETD since the number of patient days per week was $>50$ days (refer to Figure-1).

Fig. 2 Incidence rate of extensively-drug resistant Acinetobacter baumannii (XDR-AB) acquisition (number of cases who had a positive culture per 1000 patient days) in the open-bay intensive care unit (ICU) of our facility per each numerical week after enhanced terminal disinfection (ETD). N.B. -Each patient, who resided in the ICU during the corresponding numerical week, was counted once per each numerical week depending on the date of acquisition only and it was not cumulative. -The incidence rate of XDR-AB was calculated from Week 1 to Week 16 after ETD since the number of patient days per week was $>50$ days (refer to Figure-1). 
Table 2- Multivariate logistic regression of potential predictors for extensively drug-resistant Acinetobacter baumannii acquisition during the study period

\begin{tabular}{|c|c|c|}
\hline Variables & Odds Ratio (95\% Confidence Interval) & $P$ \\
\hline Wound & $3.72(0.99-13.96)$ & 0.05 \\
\hline Urinary catheter ( $\geq 5 \mathrm{~d}$ ) & $1.42(0.42-4.79)$ & 0.58 \\
\hline Mechanical ventilation & $4.99(1.76-14.15)$ & 0.002 \\
\hline \multicolumn{3}{|l|}{$\mathrm{CCl}$ score } \\
\hline 0 & $0.71(0.13-3.98)$ & 0.70 \\
\hline $5-6$ & $2.55(0.96-6.81)$ & 0.06 \\
\hline $7-8$ & $0.48(0.11-2.14)$ & 0.34 \\
\hline XDR-AB contact pressure ( $\geq 3 \mathrm{~d}$ ) & $9.86(3.65-26.64)$ & $<0.0001$ \\
\hline CAR use $(\geq 4 \mathrm{~d})$ & $2.50(0.43-14.36)$ & 0.31 \\
\hline TZP use & $2.86(0.97-8.46)$ & 0.06 \\
\hline CAR or TZP use $(\geq 4 d)$ & $0.58(0.13-2.68)$ & 0.49 \\
\hline LOS in the ICU ( $\geq 4 d)$ & $1.29(0.39-4.30)$ & 0.68 \\
\hline \multicolumn{3}{|c|}{ Number of patients staying in the ICU during each numerical week after ETD } \\
\hline Week 6 & $3.37(0.89-12.71)$ & 0.07 \\
\hline Week 7 & $6.47(1.21-34.50)$ & 0.03 \\
\hline Week 8 & $0.30(0.04-2.39)$ & 0.26 \\
\hline Week 9 & $3.23(0.59-17.75)$ & 0.18 \\
\hline Week 10 & $0.12(0.01-1.03)$ & 0.053 \\
\hline Week 11 & $9.73(1.66-57.12)$ & 0.01 \\
\hline Week 12 & $2.65(0.49-14.52)$ & 0.26 \\
\hline Week 13 & $0.14(0.02-1.24)$ & 0.08 \\
\hline Week 14 & $14.37(1.75-118.17)$ & 0.01 \\
\hline Week 15 & $1.34(0.11-15.85)$ & 0.82 \\
\hline Week 16 & $3.33(0.26-43.02)$ & 0.36 \\
\hline
\end{tabular}

Variables included in the model: Urinary catheter (reference: $<5 \mathrm{~d}$ ); Mechanical ventilation (reference: no); CCI score; XDR-AB contact pressure (reference: $<3 \mathrm{~d}$ ); CAR (reference: < $4 \mathrm{~d}$ ); TZP use (reference: no); CAR or TZP use (reference: $<4 \mathrm{~d}$ ); LOS in the ICU (Reference: $<4 \mathrm{~d}$ ); Number of patients staying in the ICU during each numerical week after ETD: Week 6 to Week 16 (reference: no)

$K E Y=C A R$ Carbapenems, CCI Charlson Comorbidity Index, $d$ days, ETD Enhanced Terminal Disinfection, ICU Intensive Care Unit, LOS: Length of Stay, TZP Piperacillin/Tazobactam, XDR-AB Extensively-drug resistant Acinetobacter bumannii

$P=0.58) ; \quad \mathrm{OR}=1.29,95 \%$ CI $(0.39-4.30), P=0.68)$; respectively] (Table 2).

\section{Numerical weeks after ETD as a potential risk factor of XDR-AB acquisition}

By bivariate analysis, Week 6 after ETD was the first to show a statistically significant difference between groups 1 and 2. Accordingly, Weeks 6 to 16 were included as potential risk factors for XDR-AB acquisition in the multivariate logistic regression. Weeks 17 to 38 after ETD were not included due to the low number of PD (< 50 days). The multivariate model showed that patients who stayed during numerical weeks 7,11 and 14 after ETD were at an increased risk of acquisition where the odds significantly increased by $6.5,9.7$ and 14.4 folds respectively ( $P=0.03,0.01$, and 0.01 , respectively) (Table 2 ). We considered numerical Week 7 after ETD as the most suitable time for ETD since it was the first numerical week with an $\mathrm{OR}>2$ (6.5) and a statistically significant p-value (0.03).

\section{Discussion}

Carbapenem-resistant A. baumanni or XDR-AB has been recently recognized by the "World Health Organization (WHO) AMR study group" as a high priority Gram-negative organism for the research and development of new antibiotics, given the limited availability of treatment options, its swiftness in developing AMR, in addition to the elevated rates of mortality due to associated infections [19]. Through current evidence, its resistance to disinfection and its endemicity around the globe has exponentially augmented the problem rendering it the nightmare of Infection Control practitioners when managing sustained outbreaks [20]. Another group of international experts developed the "TOp TEn resistant Microorganisms (TOTEM) in critical care" study 
group, which aimed to assess the global top priority organisms affecting ICU patients specifically [21]. Carbapenem-resistant A. baumannii was again classified as the top critical organism thus coinciding with the WHO priority list [21].

Many critical care and burn units of acute care facilities in the Middle East and North Africa region have become endemic with XDR-AB [22]. In the current study, out of 335 patients admitted to our ICU, 13\% acquired XDR-AB during their stay and the overall incidence reached 14.6 cases/1000 ICU days. Other studies from Lebanon and the region reported different burdens of XDR-AB in critical care units. A prospective study conducted over 7 years form 2007 to 2014 at a large university hospital in Beirut showed that the a rate of XDR$\mathrm{AB}$ colonization pressure was 315.4 cases/1000 ICU patient-days [3]. Another matched case-control study from a specialist hospital in the Kingdom of Saudi Arabia between January and August 2012, assessing potential risk factors for XDR-AB acquisition, showed that the proportion of ICU patients who harbored XDR-AB during their stay reached 33\% (66/198 patients) [23]. These figures indicate in our geographical region that XDR-AB is rampant in critical care units.

Infections caused by A. baumanii in general and especially among critically ill patients are associated with high morbidity and mortality [24]. To be able to reduce this high mortality rate, a multidisciplinary approach is essential, including early detection and surveillance for acquisition, strategies for patient risk factor identification, antimicrobial stewardship interventions, and tight adherence to standardized infection control practices $[2$, 25-28].

Risk factor identification and mitigation are critical pillars in decreasing the transmission of XDR-AB [2]. Our study showed that contact pressure of 3 or more days with other patients who harbored XDR-AB was an independent risk factor for acquisition of the same organism, and it significantly increased the odds of horizontal acquisition by almost 10 folds. This can be explained by the fact that the open-bay ICU is one big room and all bed occupants are like "roommates". It has been also well documented that patients admitted to ICU beds of prior occupants who harbored bacterial pathogens are at an elevated risk of acquiring the same organism, i.e. vertical transmission of the organism [29]. In a multicenter matched case-control study that evaluated the association between having a prior bed occupant or roommate with a positive culture and subsequent infection with the same organism, the risk of infection increased by 6 folds in case of the prior bed occupant (vertical transmission) and 5 times in case of the roommate (horizontal transmission) [30]. With time, transmission of XDR-AB occurring in both directions, vertical and horizontal, will absolutely increase and one will lead to the other in an open-bay ICU. In this setting, ICU evacuation and ETD have been practiced to potentially stem the vertical transmission and break the vicious cycle.

Our results further showed that patients who were administered carbapenems for more than 4 days or piperacillin-tazobactam were more likely to acquire XDR-AB during their hospital stay. Antimicrobial stewardship programs play an important role in sparing the use of carbapenems and other broad-spectrum antibiotics [27]. Their extensive use has been positively correlated with an increasing incidence of XDR-AB [31], while restricting their consumption has led to an important reduction in its incidence [25, 26, 28]. The proper selection of broad-spectrum antibiotics for the empiric treatment of infections is based on the institutional epidemiology of AMR, along with an appropriate duration and de-escalation of therapy once antibiograms are available.

In general, risk factors for XDR-AB acquisition may vary from one ICU to the other due to differences in applying standard precautions, compliance to hand hygiene, distance between beds, type of ICU whether open-bay or single-room [2, 32]. On the other hand, risk factors including the use of invasive devices, treatment with broad-spectrum antibiotics, length of stay in ICU and contact pressure are commonly reported in several studies [2, 3, 23, 33].

Regarding infection prevention, the presence of robust programs is crucial in order to help curb the spread of XDR-AB throughout healthcare systems. Patient cohorting, improved hand hygiene, regular environmental cleaning and disinfection, in addition to novel non-touch techniques used in ETD have succeeded in reducing nosocomial infection rates and controlling outbreaks of XDR-AB [25, 26]. Contactless automated decontamination technologies include aerosol or vaporized $\mathrm{H}_{2} \mathrm{O}_{2}$ and mobile continuous germicidal ultraviolet light (UVC). Yet, the success of ETD, which is only one part of the puzzle, depends on human factors such as training and compliance of nursing and environmental services staff, as well as accessibility of all inanimate surfaces in the unit. The quality of surface disinfection is highlighted by a study that proved surface contamination with epidemiologically important pathogens owed to a failure to practice thorough cleaning and disinfection rather than a faulty product or procedure [34].

Based on our findings, XDR-AB acquisition followed an ascending trend as a function of numerical weeks after ETD $(P=0.03)$. This implied that more patients acquired XDR-AB with time after ETD due to the waning effect of the procedure. The complete evacuation of the ICU and admitting patients who are not infected or colonized with XDR-AB decreased the contact pressure 
during the early numerical weeks after ETD, which is a very strong factor of $\mathrm{XDR}-\mathrm{AB}$ acquisition as mentioned previously. The effect of the contactless modality of disinfection using aerosolized $\mathrm{H}_{2} \mathrm{O}_{2}$ could be explained as such. First, the physical and biochemical properties of the aerosolized $\mathrm{H}_{2} \mathrm{O}_{2}$ particles enable it to reach and potentially decontaminate surfaces that are usually inaccessible by manual techniques [35-37]. Second, $\mathrm{H}_{2} \mathrm{O}_{2}$ could synergistically improve the effect of other common agents used in manual disinfection, such as quaternary-ammonium containing products (used in our case), when applied to cleaned surfaces with potential residual XDR-AB contamination [35-37].

A study from the United Kingdom investigated the efficacy of terminal disinfection using different operating concentrations of vaporized hydrogen peroxide on methicillin-resistant Staphylococcus aureus (MRSA), Klebsiella pneumoniae and Clostridium difficile persistence on single isolation room surfaces after patient discharge [38]. Investigators artificially contaminated highfrequency-touch surfaces with these organisms, where the sites were sampled with contact plates before and after hydrogen peroxide fumigation [38]. After manual disinfection only, more than $90 \%$ of the sites were still contaminated with these organisms, with high bacterial count present on floors, bed control panels, and nurse call buttons [38]. Enhanced disinfection with hydrogen peroxide achieved an approximately $5 \log 10$ reduction in C. difficile spores on contact plates and an approximately $6 \log 10$ reduction in MRSA/K. pneumoniae colony forming units on contact plates in all tested areas [38].

The direct effect of ETD in general was recently elucidated in the Benefits of Enhanced Terminal Room (BETR) Disinfection study, a prospective clusterrandomized crossover trial [14]. This study assessed 3 different enhanced methods of room disinfection (bleach, quaternary ammonium-containing product with disinfecting UV-C, and bleach with UV-C compared to a standard disinfection method with quaternary ammonium-containing product only (control) [14]. Results showed that enhanced methods of disinfection overcame limitations of standard disinfection strategies and thus could be potential strategies to reduce the risk of acquisition of multidrug-resistant organisms and Clostridium difficile [14]. This trial has shown the efficacy of using UV-C as a non-touch strategy for enhanced disinfection, but not $\mathrm{H}_{2} \mathrm{O}_{2}$. Limited data are available on the activity of aerosolized $\mathrm{H}_{2} \mathrm{O}_{2}$ based on laboratory findings or evaluation of experimentally contaminated surfaces in hospitals [15]. An aerosolized $\mathrm{H}_{2} \mathrm{O}_{2}$ system was capable of eradicating methicillin-resistant Staphylococcus aureus and A. baumannii on open hospital room surfaces; however, it was not effective in closed or semiclosed areas like inside a drawer [36]. On the other hand, Blazejewski and colleagues reported results of a prospective crossover study in five medical and surgical ICUs located in a single tertiary care hospital, which examined the impact of ETD using $\mathrm{H}_{2} \mathrm{O}_{2}$ on environmental contamination with multidrug-resistant organisms including imipenemresistant $A$. baumannii [39]. In this study, target rooms ( $n=182)$ underwent routine terminal cleaning with a quaternary ammonium compound and bleach. Then, they were disinfected by either $\mathrm{H}_{2} \mathrm{O}_{2}$ vapor or aerosolized $\mathrm{H}_{2} \mathrm{O}_{2}$ combined with peracetic acid during 6 weeks with a switch to the other method for another 6 weeks [39]. Environmental sampling of 8 high-touch surfaces was performed in each room at 3 time points: (1) after patient discharge, (2) after routine terminal cleaning, and (3) after ETD. First after patient discharge, 15/ $182(8 \%)$ rooms were contaminated with at least one multi-drug resistant organism [39]. Then, routine terminal cleaning reduced environmental contamination with multi-drug resistant organisms from 8 to 6\% (11/ 182 rooms), albeit insignificantly $(P=0.371)$ [14]. Yet, investigators observed a significant reduction in environmental contamination with multi-drug resistant organisms from 11/182 rooms (6\%) (after routine terminal cleaning) to $1 / 182$ rooms $(0.6 \%)$ after ETD using $\mathrm{H}_{2} \mathrm{O}_{2}(P=0.004)$. Interestingly, both studied techniques (aerosolized and vapor $\mathrm{H}_{2} \mathrm{O}_{2}$ ) showed similar disinfection efficacy [39]. The evidence provided in the aforementioned studies could be used to interpret our results, through highlighting the disinfection efficacy of ETD with $\mathrm{H}_{2} \mathrm{O}_{2}$, in addition to the effect of decreasing contact pressure after ICU evacuation as mentioned earlier. More robust studies on room decontamination should be performed with specific $\mathrm{H}_{2} \mathrm{O}_{2}$ formulations and related ETD techniques, and these should be compared to other standardized and reliable methods.

As we previously mentioned, ETD necessitated the evacuation and closure of our open-bay ICU for at least $24 \mathrm{~h}$. It was not feasible on a regular basis after the discharge of each infected or colonized patient with XDR$\mathrm{AB}$ from the unit. Such intervention is costly regarding the loss of ICU bed occupancy during the time of unit closure. On the other hand, XDR-AB acquisition exerts an economic burden on the hospital, not to mention the associated morbidity and mortality [40]. Finding the optimal timing for ETD would help prevent sustained transmission on one the hand, and unnecessary evacuation and loss of bed occupancy on the other hand. As per the multivariate regression analysis in our cohort, the first numerical week that considerably increased the odds of XDR-AB acquisition with respect to control was 'Week 7' after ETD. The chance that a patient staying in the ICU during 'Week 7' to have acquired XDR-AB was 6.5 times significantly higher than not to acquire it. So, 
we recommend performing ETD with aerosolized $\mathrm{H}_{2} \mathrm{O}_{2}$ every 7 calendar weeks in our open-bay ICU as part of an infection control bundle, in order to limit the increasing rate of $\mathrm{XDR}-\mathrm{AB}$ acquisition with time.

The increasing risk of XDR-AB acquisition with time after ETD in an open-bay ICU makes this design a questionable situation. Separate rooms or cubicles where ETD can be performed after the stay of each colonized or infected patient might be the preferable architecture of an ICU in the era of virulent XDR organism transmission, along with the paucity of effective antimicrobials [12]. Halaby et al. successfully described the implementation of a single room policy in their ICU compared to a previous open-bay design. This shift resulted in a clear and sustained decrease in the prevalence of the multidrug resistant Gram-negative bacteria, keeping in mind that no significant changes in variables including bed occupancy and numbers of patient admissions was documented during the study period [32]. In 2018, the revised US guidelines of hospital construction provided a new recommendation that critical care units be designed on a single-room basis except for neonatal ICUs [41]. An exception is provided for renovation of patient rooms or cubicles for single-patient use provided they have a minimum clear floor area of 150 square feet [41].

\section{Limitations and strengths}

One major limitation in this study is that our finding regarding the optimal timing of ETD is specific to our open-bay ICU and may not be applicable to other hospital ICUs. Factors affecting this result are the degree of compliance with hand hygiene requirements, the extent of application of patient isolation and standard precautions policies, nurse-to-patient ratios, bed-to-bed distance, and the endemicity of XDR organisms other than A. baumannii. Nevertheless, an attempt to define the most appropriate timing for ETD in a limited-resource setting is a useful roadmap that would assist other researchers build upon and other practitioners plan ETD schedules in their facilities. On the other hand, our findings highlight the need to shift from an open-bay ICU to individual rooms in this era of XDR organisms. Our retrospective study design is another drawback, where a prospective randomized approach with a crossover design would come up with more reliable results, while controlling for potential confounders. Another limitation is the lack of proof for the activity of the $\mathrm{H}_{2} \mathrm{O}_{2}$ decontamination on the quantitative load of $\mathrm{A}$. baumannii in the inanimate environment like it has been demonstrated for other organisms including K. pneumoniae, MRSA and C. difficile. This opens the door to further research regarding the optimal ways to enhance cleaning and disinfecting surfaces contaminated with A. baumannii.

\section{Conclusion}

Contact pressure, indwelling urinary catheters, mechanical ventilation, presence of a wound along with the use of broad spectrum antimicrobials are risk factors for the acquisition of XDR-AB in our facility. Temporary ICU closure along with ETD using aerosolized $\mathrm{H}_{2} \mathrm{O}_{2}$ and Silver cations decreased the rate of XDR-AB acquisition, yet this effect fades away with time. The ETD was shown to be most efficiently done when repeated every 7 calendar weeks in our open-bay ICU as part of an infection control bundle, in order to limit the increasing rate of $\mathrm{XDR}-\mathrm{AB}$ acquisition with time while most optimally utilizing costly hospital resources. However, traditional cleaning methods should not be relaxed or abandoned even if ETD is regularly performed, emphasizing on the importance on properly using surface disinfectants to remove biofilms. Isolated patient areas with the possibility of independent enhanced cubicle disinfection after discharge of each colonized or infected patient would preferably replace open-bay ICUs in this era of spreading antibiotic-resistant organisms.

\section{Abbreviations}

AMR: Antimicrobial Resistance; APACHE II: Acute Physiologic Assessment and Chronic Health Evaluation II; BiPAP: Bilevel Positive Airway Pressure; CCI: Charlson Comorbidity Index; CDC: Centers for Disease Control and Prevention; Cl: Confidence Interval; CLSI: Clinical and Laboratory Standards Institute; ETD: Enhanced Terminal Disinfection; $\mathrm{H}_{2} \mathrm{O}_{2}$ : Hydrogen Peroxide; ICU: Intensive Care Unit; MGH: Makassed General Hospital; NW: Numerical Weeks; OR: Odds Ratio; PD: Patient Days; RFA: Risk Factors of Acquisition; SPSS: Statistical Package for the Social Sciences; TOTEM: TOp TEn resistant Microorganisms; WHO: World Health Organization; XDR-AB: Extensively DrugResistant Acinetobacter baumannii

\section{Acknowledgements \\ None.}

\section{Authors' contributions}

RM was responsible for study conception, result analysis and drafting of the manuscript. HT was responsible for study conception and result analysis. MJ, RK, HK, HH, SH, LA, RL, NS contributed to data collection and analysis. DA performed data analysis and contributed to drafting and reviewing the final version of the manuscript. TJ was responsible for the microbiological analysis. NH edited the final version of the manuscript. All authors read and approved the final version of the manuscript.

\section{Funding \\ None.}

\section{Availability of data and materials}

The data that support the findings of this study are available from Makassed General Hospital but restrictions apply to the availability of these data, which were used under license for the current study, and so are not publicly available. Data are however available from the authors upon reasonable request and with permission of Makassed General Hospital.

\section{Ethics approval and consent to participate}

The institutional review board (IRB) committee of Makassed General Hospital, Beirut, Lebanon, granted this study ethical approval. The IRB committee waived the requirement of informed consent from patients due to the retrospective nature of this study. During the data collection phase, only subject case numbers were included. At a later stage, a different number was assigned to each of our cases to safeguard subject privacy. The contributing authors only performed data entry and analysis as well as the drafting of the manuscript. 


\section{Consent for publication}

Not applicable.

\section{Competing interests}

The authors declare that they have no competing interests.

\section{Author details}

'Division of Infectious Diseases, Department of Internal Medicine, Makassed General Hospital, Beirut, Lebanon. ${ }^{2}$ Division of Infectious Diseases, Hôtel Dieu de France, Beirut, Lebanon. ${ }^{3}$ Department of Internal Medicine, American University of Beirut, Beirut, Lebanon. ${ }^{4}$ School of Pharmacy, Beirut Arab University, Beirut, Lebanon. ${ }^{5}$ Pharmacy Department, Makassed General Hospital, Beirut, Lebanon. ${ }^{6}$ Nursing Department, Makassed General Hospital, Beirut, Lebanon. ${ }^{7}$ Department of Internal Medicine, Makassed General Hospital, Beirut, Lebanon. ${ }^{8}$ Faculty of Arts and Sciences, American University of Beirut, Beirut, Lebanon. ${ }^{9}$ Department of Laboratory Medicine, Makassed General Hospital, Beirut, Lebanon. ${ }^{10}$ College of Human Ecology, Cornell University, Ithaca, NY 14853, USA. " Associate Professor of Infectious Disease and Residency Program Director, Internal Medicine, Central Michigan University, Saginaw, MI 48602, USA.

Received: 15 January 2020 Accepted: 2 July 2020

Published online: 14 July 2020

\section{References}

1. WHO. Global antimicrobial resistance surveillance system (GLASS) report; 2018. http://www.who.int/glass/resources/publications/earlyimplementation-report/en/ (Accessed 4 Jan 2020).

2. Moghnieh R, Siblani L, Ghadban D, et al. Extensively drug-resistant Acinetobacter baumannii in a Lebanese intensive care unit: risk factors for acquisition and determination of a colonization score. J Hosp Infect. 2016; 92(1):47-53.

3. Kanafani ZA, Zahreddine $N$, Tayyar $R$, et al. Multi-drug resistant Acinetobacter species: a seven-year experience from a tertiary care center in Lebanon. Antimicrob Resist Infect Control. 2018;7(1):9.

4. Ballouz T, Aridi J, Afif C, et al. Risk factors, clinical presentation, and outcome of Acinetobacter baumannii bacteremia. Front Cell Infect Microbiol. 2017;7:156.

5. Al Atrouni A, Hamze M, Jisr T, et al. Wide spread of OXA-23-producing carbapenem-resistant Acinetobacter baumannii belonging to clonal complex II in different hospitals in Lebanon. Int J Infect Dis. 2016;52:29-36.

6. Moghnieh R, Araj GF, Awad L, et al. A compilation of antimicrobial susceptibility data from a network of 13 Lebanese hospitals reflecting the national situation during 2015-2016. Antimicrob Resist Infect Control. 2019;8(1):41.

7. Abdallah D, El Mchad H, Moghnieh R. Pandrug-resistant Acinetobacter baumannii infections: case series, contributing factors, outcomes and available treatment options. Clinical cases in microbiology and infectious diseases. Ghassan Matar, New York: Elsevier, 2017. 47-57.

8. Fraimow HS, Tsigrelis C. Antimicrobial resistance in the intensive care unit: mechanisms, epidemiology, and management of specific resistant pathogens. Crit Care Clin. 2011;27(1):163-205.

9. Clark NM, Zhanel GG, Lynch JP III. Emergence of antimicrobial resistance among Acinetobacter species: a global threat. Curr Opin Crit Care. 2016; 22(5):491-9.

10. Kramer A, Schwebke I, Kampf G. How long do nosocomial pathogens persist on inanimate surfaces? A systematic review. BMC Infect Dis. 2006; 6(1):130.

11. Chamoun K, Farah M, Araj G, et al. Surveillance of antimicrobial resistance in Lebanese hospitals: retrospective nationwide compiled data. Int J Infect Dis. 2016;46:64-70

12. Otter JA, Yezli S, Salkeld JA, et al. Evidence that contaminated surfaces contribute to the transmission of hospital pathogens and an overview of strategies to address contaminated surfaces in hospital settings. Am J Infect Control. 2013;41(5):S6-11.

13. Weber $D J$, Anderson $D$, Rutala WA. The role of the surface environment in healthcare-associated infections. Curr Opin Infect Dis. 2013;26(4):338-44.

14. Anderson DJ, Chen LF, Weber DJ, et al. Enhanced terminal room disinfection and acquisition and infection caused by multidrug-resistant organisms and Clostridium difficile (the benefits of enhanced terminal room disinfection study): a cluster-randomised, multicentre, crossover study. Lancet. 2017;389(10071):805-14.

15. Weber DJ, Rutala WA, Anderson DJ, et al. Effectiveness of ultraviolet devices and hydrogen peroxide systems for terminal room decontamination: focus on clinical trials. Am J Infect Control. 2016;44(5):e77-84.

16. Siegel JD, Rhinehart $\mathrm{E}$, Jackson $\mathrm{M}$, et al. Management of multidrug-resistant organisms in health care settings, 2006. Am J Infect Control. 2007;35(10): S165-93.

17. CLSI. Performance standards for antimicrobial susceptibility testing; twentyeighth informational supplement. In: CLSI document M100-S28. Wayne: Clinical and Laboratory Standards Institute; 2018.

18. Magiorakos AP, Srinivasan A, Carey RB, et al. Multidrug-resistant, extensively drug-resistant and pandrug-resistant bacteria: an international expert proposal for interim standard definitions for acquired resistance. Clin Microbiol Infect. 2012;18:268-81.

19. Tacconelli E, Carrara E, Savoldi A, et al. Discovery, research, and development of new antibiotics: the WHO priority list of antibiotic-resistant bacteria and tuberculosis. Lancet Infect Dis. 2018;18(3):318-27.

20. Kenner L, Wallace B, Strelczyk K, et al. Pan-drug-resistant Acinetobacter baumannii: the new MRSA? Nurs Crit Care. 2011;6(5):32-7.

21. Rello J, Eshwara VK, Lagunes $L$, et al. A global priority list of the TOp TEn resistant microorganisms (TOTEM) study at intensive care: a prioritization exercise based on multi-criteria decision analysis. Eur I Clin Microbiol Infect Dis. 2019;38(2):319-23.

22. Moghnieh RA, Kanafani ZA, Tabaja HZ, et al. Epidemiology of common resistant bacterial pathogens in the countries of the Arab league. Lancet Infect Dis. 2018;18(12):e379-94.

23. Al-Gethamy MM, Faidah HS, Adetunji HA, et al. Risk factors associated with multi-drug-resistant Acinetobacter baumannii nosocomial infections at a tertiary care hospital in Makkah, Saudi Arabia-a matched case-control study. J Int Med Res. 2017:45(3):1181-9.

24. Falagas ME, Bliziotis IA, Siempos II. Attributable mortality of Acinetobacter baumannii infections in critically ill patients: a systematic review of matched cohort and case-control studies. Crit Care. 2006;10(2):R48.

25. Chen CH, Lin LC, Chang YJ, et al. Infection control programs and antibiotic control programs to limit transmission of multi-drug resistant Acinetobacter baumannii infections: evolution of old problems and new challenges for institutes. Int J Environ Res Public Health. 2015;12(8):8871-82.

26. Cheon S, Kim MJ, Yun SJ, et al. Controlling endemic multidrug-resistant Acinetobacter baumannii in intensive care units using antimicrobial stewardship and infection control. Korean J Intern Med. 2016;31(2):367.

27. Wenzler E, Goff DA, Humphries R, et al. Anticipating the unpredictable: a review of antimicrobial stewardship and Acinetobacter infections. Infect Dis Ther. 2017;6(2):149-72.

28. Chamieh A, Nawfal TD, Ballouz T, et al. Control and elimination of extensively drug-resistant Acinetobacter baumanii in an intensive care unit. Emerg Infect Dis. 2019;25(10):1928.

29. Cohen B, Cohen CC, Løyland B. Transmission of healthcare-associated infections from roommates and prior room occupants: a systematic review. Clin Epidemiol. 2017;9:297-310.

30. Cohen B, Liu J, Cohen AR, et al. Association between healthcare-associated infection and exposure to hospital roommates and previous bed occupants with the same organism. Infect Control Hosp Epidemiol. 2018;39(5):541-6.

31. Lee HS, Loh YX, Lee JJ, et al. Antimicrobial consumption and resistance in five gram-negative bacterial species in a hospital from 2003 to 2011. J Microbiol Immunol Infect. 2015;48:647-54.

32. Halaby T, Al Naiemi N, Beishuizen B, et al. Impact of single room design on the spread of multi-drug resistant bacteria in an intensive care unit. Antimicrob Resist Infect Control. 2017:6(1):117.

33. Ibrahim ME. Prevalence of Acinetobacter baumannii in Saudi Arabia: risk factors, antimicrobial resistance patterns and mechanisms of carbapenem resistance. Ann Clin Microbiol Antimicrob. 2019;18(1):1.

34. Hota B, Blom DW, Lyle EA, et al. Interventional evaluation of environmental contamination by vancomycin-resistant enterococci: failure of personnel, product or procedure? J Hosp Infect. 2009;71:123-31.

35. Barbut $F$, Menuet $D$, Verachten $M$, et al. Comparison of the efficacy of a hydrogen peroxide dry-mist disinfection system and sodium hypochlorite solution for eradication of Clostridium difficile spores. Infect Control Hosp Epidemiol. 2009;30(6):507-14.

36. Piskin N, Celebi G, Kulah C, et al. Activity of a dry mist-generated hydrogen peroxide disinfection system against methicillin-resistant Staphylococcus 
aureus and Acinetobacter baumannii. Am J Infect Control. 2011;39(9):

757-62.

37. Mosci D, Marmo GW, Sciolino L, et al. Automatic environmental disinfection with hydrogen peroxide and silver ions versus manual environmental disinfection with sodium hypochlorite: a multicentre randomized beforeand-after trial. J Hosp Infect. 2017;97(2):175-9.

38. Ali S, Muzslay M, Bruce M, Jeanes A, Moore G, Wilson AP. Efficacy of two hydrogen peroxide vapour aerial decontamination systems for enhanced disinfection of meticillin-resistant Staphylococcus aureus, Klebsiella pneumoniae and Clostridium difficile in single isolation rooms. J Hosp Infect. 2016;93(1):70-7.

39. Blazejewski C, Wallet $F$, Rouze $A$, et al. Efficiency of hydrogen peroxide in improving disinfection of ICU rooms. Crit Care. 2015;19:30.

40. Lee BY, McGlone SM, Doi Y, et al. Economic impact of Acinetobacter baumannii infection in the intensive care unit. Infect Control Hosp Epidemiol. 2010;31(10):1087-9.

41. The Facility Guidelines Institute. Guidelines for design and construction of hospitals. St. Louis: Facility Guidelines Institute; 2018.

\section{Publisher's Note}

Springer Nature remains neutral with regard to jurisdictional claims in published maps and institutional affiliations.

Ready to submit your research? Choose BMC and benefit from:

- fast, convenient online submission

- thorough peer review by experienced researchers in your field

- rapid publication on acceptance

- support for research data, including large and complex data types

- gold Open Access which fosters wider collaboration and increased citations

- maximum visibility for your research: over $100 \mathrm{M}$ website views per year

At $\mathrm{BMC}$, research is always in progress.

Learn more biomedcentral.com/submissions 